\title{
Implementation of NOGIE and NOWGIE for Human Skin Detection
}

\author{
M. Omer Aftab, Junaid Javed, M. Bilal, Arfa Hassan \\ Department of Computer Science \\ Lahore Garrison University, \\ Lahore, Pakistan.
}

\author{
M. Adnan Khan \\ Department of Computer Science \\ NCBA\&E, \\ Lahore, Pakistan.
}

\begin{abstract}
The Digital image processing is one of the most widely implemented fields worldwide. The most applied applications of digital image processing are facial recognition, finger print recognition, medical imaging, law enforcement, cyber-crime investigation, identification of various diseases and criminals, etc. The subject to be discussed in this article is skin detection. Skin detection has solved many serious problems related to digital image process. It is one of the main features in making an intelligent image processing system. The proposed methodology conducts an improved and well enhanced skin detection, the skin and non-skin parts are divided from an input image or video, noise is removed, HSV is applied which also acts as a color model that generates more better results in accordance to RGB or $\mathrm{YCbCr}$ for skin and face identification. The algorithms, NOGIE (Noise Object Global Image Enhancement) and NOWGIE (Noise Object with Global Image Enhancement) are applied separately on the input and the results can be compared for better perception and understanding of the applied skin detection techniques, the skin parts are highlighted as "White" while the Non-skin parts are highlighted as "Black". The results are different NOWGIE gives better results than the NOGIE due to the image enhancement technique. This methodology is subjected to be implemented in special security drones for the identification of suspects, terrorists and spy's the algorithms provides the ability to detect humans from a non-skin background making an autonomous and excellent security system.
\end{abstract}

Keywords-Skin detection; Digital Image Processing (DIP); Noise Object Global Image Enhancement (NOGIE); Noise Object with Global Image Enhancement (NOWGIE); Hue Saturation and Value (HSV); RGB

\section{INTRODUCTION}

The digital image processing is one of the most widely implemented fields throughout and is a big step towards autonomy. The world is comprising of a tremendous number of applications working on the very foundation of digital image processing that one is surrounded by that cannot be neglected. The skin detection is a branch of digital image processing that have gained quite much importance and is continued to do so. The rapid technological growth causing the implementation of skin detection widely so much making it a complete domain inherited with numerous contraptions, like fingerprint recognition, face detection and recognition, lip reading, pattern recognition, artificial intelligence and much more [1]. Currently it is also subjected to be playing a vital role in various security systems for the reduction in criminal activities, better law enforcement like intelligent traffic video surveillance that uses the skin detection techniques for human facial recognition to identify criminals etc. [2]. The question is how does it work? An intelligent skin detection system should be able to detect human skin when it is provided with some input image or video. If it is accompanied by an image comprising a human, some animals and trees etc. the system must identify the human it detects the human skin by stratification of human skin color and texture as the human skin is not specifically geometric, skin detection leads to facial recognition [3]. The system works by categorizing via color segmentation of the skin and non-skin parts ignoring the nonskin part. On the skin part it is able to identify the human skin as it is provided by the (Hue Saturation and value) HSV as an input. The HSV acts as a color model that is more efficient than the RGB, the procedure requires background knowledge about the objects regarding within the image [4]. The color segmentation helps to skim and identify the skin from which can recognize the faces, finger prints and other human body parts.

In this article the proposed methodology is actually an upgraded version of the previous work done that was the application of GIE and without GIE techniques for skin detection [5]. This enhanced version is the application of the NOGIE and NOWGIE algorithms to detect human skin from an image or video. Both of the methodologies basically work on the same principle of human skin detection. In this methodology, These algorithms are very different from the previous and other various skin detection techniques as it promotes autonomy and more improved results. NOGIE stands for (Noise Object Global Image Enhancement) and NOWGIE stands for (Noise Object With Global Image Enhancement). Both of the algorithms are quite related to the GIE and without GIE terms, but these algorithms are applied step wise and gives a more enhanced output for the detection of human skin providing better results. On the input image or video first the NOGIE algorithm is applied after that the NOWGIE algorithm is applied and their results are compared at the end. The application of this methodology is via MATLAB, an image or video is read and then the noise is removed after that object is detected the HSV value is inserted in the algorithm here the HSV acts as a membership function that varies and continue to change it until the value is set for the desired results. After the skin is detected, same procedure is repeated on the input, but equalization is implemented after the object detection. This methodology is subjected to be 
deployed in special security drones for the identification of criminal suspects, terrorists or spy's. For example, if a spy or terrorist of an enemy have crossed the border or is in a sensitive area these special drones will be released to search the targeted area where the spy, terrorist is expected to hide. The drones will start to look up and will be able to detect the suspect from a non-skin background with ease. Further the drones can be programmed to perform some action after the identification of a spy like to turn on the alarms, call the security teams, etc. making a highly intelligent autonomous fool-proof security system.

\section{LITERATURE REVIEW}

After studying and reviewing a lot of research papers relative to skin detection there are various techniques being applied in accordance to detect human skin. The HSV is far better than the regular RGB. First is to acquire the optimum results the RGB space is converted into the HSV space, HSV color space is independent of the three-color components of the RGB color space. Then calculate the differences in the Histograms of successive video frames on each color component respectively the calculated Histogram difference is the base for the feature detection, this fuzzy logic based human skin detection technique is proved to be very satisfactory, but the Interpolation methodology have proven to be far better [6], [7]. Applying the skin segmentation along with the fuzzy logic is another technique where the whole frame comprising skin pixels is divided into two parts one is the fuzzy part [6] and the other is skin segmentation, training the system requires images with face and non-face here the image pixels are read in row segments to form the column segments the images consisting of face the system takes then as 1 while the comprising of no face the system takes it as a T$\mathrm{S}$ model based fuzzy training is implemented and the fuzzy function comprising weights are learnt via algorithm training the system requires multiple images of different pixel values, distances and sizes, 69 face and 56 faceless images were provided for system training for accurate results in detecting faces at instant speed [8]. A simple method is applied for the acquisition of skin pixels from RGB images consisting of facial constraints, such RGB image is taken as an input then techniques are applied for the detection of nose, the color pixels of nose's skin tone is extracted the nose is considered as a main region for the identification of the same skin pixels from the facial regions after that skin segmentation is performed and histogram model is constructed by applying fusion strategy via Gaussian Model, the results are obtained and compared both Gaussian model and fusion strategy gives good results but the Fusion strategy provides the best output [9]. The methodology works for the acquisition of facial features from images with faces within automatically by the selection of convolutional neural network (CNN) that is already trained the $\mathrm{CNN}$ is one of the most used type of artificial neural network for digital image processing comprising of multi-layer architecture, the trained CNN when provided with an image it divides the pixels and tend to identify the skin toned pixels that leads to the identification of facial structure, this methodology is capable of matching a face when young and when old as the skin tend to get older with time but this technique not only recognize the human face but also identifies a face when young and when it gets older by memorizing the facial constraints of a younger face and calculates the age distance with the same older face of singleton and image data set efficiently [10].

\section{PRoblem Statement}

The problem that arises in the lack of accuracy for skin detection and also the mistakes, bugs in the algorithms that affect the output and can also alter the results. If the skin detection is accurate the output displayed is often not very clear or enhanced. In the proposed method the algorithm does an accurate skin detection along with enhancement of the output image that makes it more visible and clearer to understand. Apart from the facial or finger print recognition the skin detection is also applied in medical sciences for the identification of various skin diseases, infections etc. This makes it more complicated for the exact identification of any skin disease. Because if not can cause problems and lead to false treatment and medication as the system is unable to get a proper input so how it will be able to generate an accurate output.

\section{PROPOSED METHOD}

In this paper the proposed methodology is a complete and improved skin detection technique, the algorithms are selfsufficient to detect human skin from an image and video. Fig. 1 shows a complete diagrammatic depiction of the proposed methodology. When a video is provided as the input the algorithms work by first reading the video and then the framing is done, still frames are selected and the methodology is implemented the procedure is same for an image, images are already static. The detection of a certain object is via cascade object detector, It divides the input into two parts skin and non-skin parts, the skin parts are highlighted as "White" while the non-skin parts as "Black". If a video is provided as input it reads the video frame by frame it selects a static frame apply the skin detection because during a video a change in body posture or face angle it will not work. Fig. 2 shows a noisy image, the algorithm implements a few steps in the noise removal from the input image or the static frame from the video. Fig. 3 shows an image after the noise removal while the Fig. 4 represents the steps done for the removal of noise from the input image. Fig. 5 shows the next step that the face from the input image or video is searched via a function known as the [vision Histogram Based Tracker] the function acts as an object for the skin detection after that it detect the facial constraints like the eyes, nose, skin tone, skin texture this allows it to uniquely identify a human with respect to the non-skin background, but if talk about the nose, it initiates with more precise skin detection as the nose is a skin part with respective skin pixels in background leads to increase in the accuracy of face detection. Fig. 6 briefs about the application of equalization for the NOWGIE algorithm this procedure is also followed by the histogram plotting that is shown in the Fig. 7 that a histogram will be plotted with respect to the input image and the resultant image of skin detection. Fig. 8 shows the original image. Fig. 9 explains the acquisition of skin tone by the HSV that acts as a membership function for the input video and image, the algorithm detects the skin and the HSV values of the input image. Further the next step is the light 
compensation of the image shown in Fig. 10. Fig. 11 depicts the skin detection via NOGIE, the skin-parts in the input are represented as "White" while the non-skin parts are represented as "Black" while Fig. 12 depicts the skin detection via NOWGIE.

A. Algorithms

The algorithms of proposed methods are as following:

1) NOGIE
a) Image/ Video acquisition
b) Noise removal
c) Object detection.
d) $\mathrm{HSV}$
e) Skin detection

2) NOWGIE
a) Image/ Video acquisition
b) Noise removal
c) Object detection.
d) Enhancement
e) HSV
f) Skin detection Selection.

B. Flow Chart

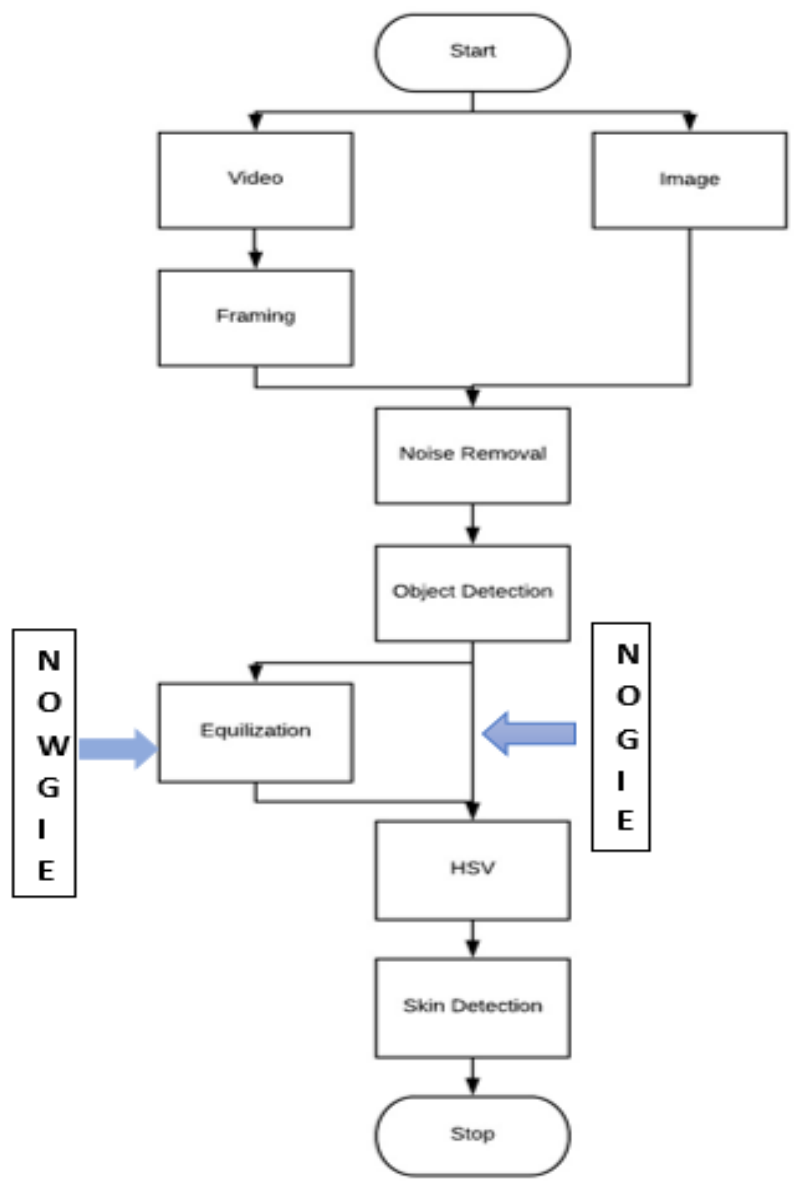

Fig. 1. Flow chart of proposed methodology.
V. Simulation AND Results

For simulation and results MATLAB R2017a is used. The simulation results are as following:
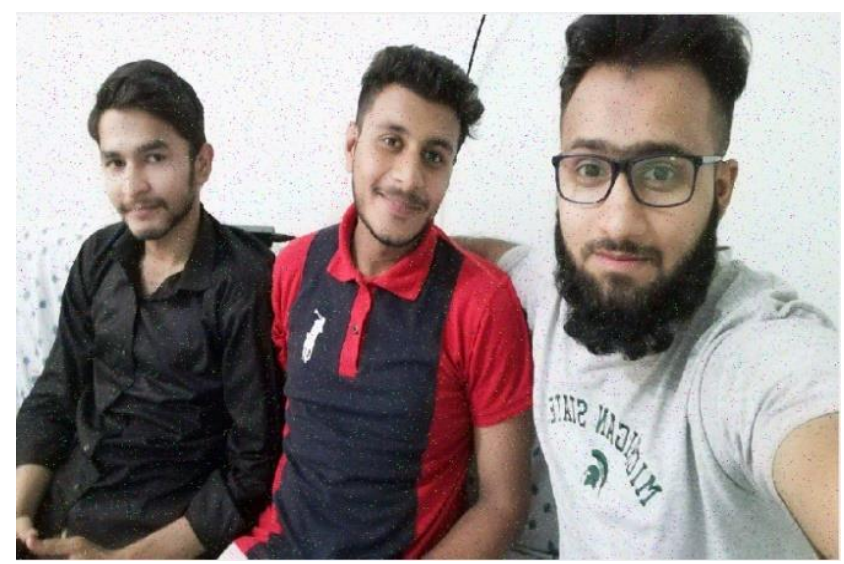

Fig. 2. Noisy image.

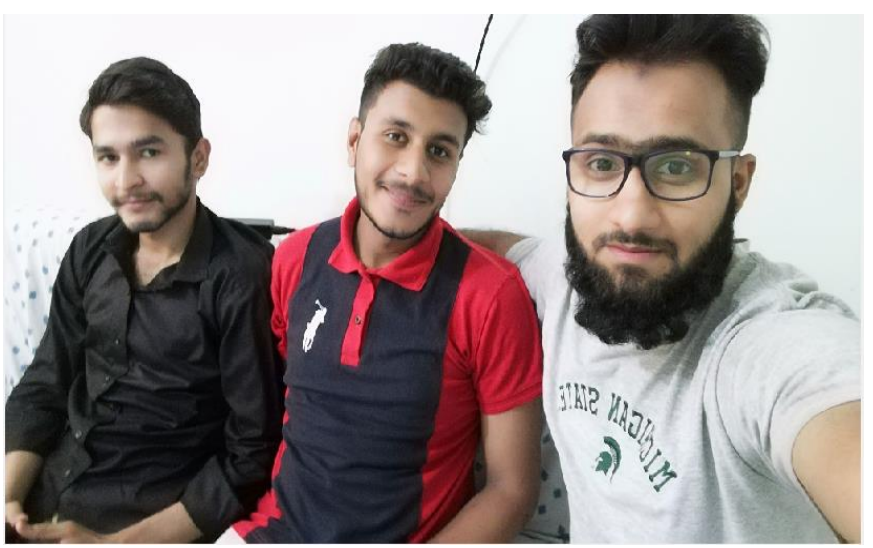

Fig. 3. Image after noise removal.

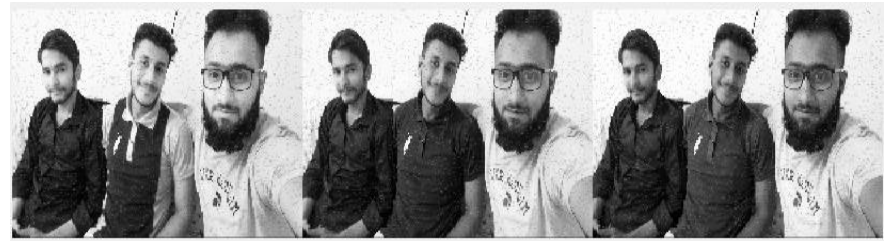

Fig. 4. Steps of noise removal.

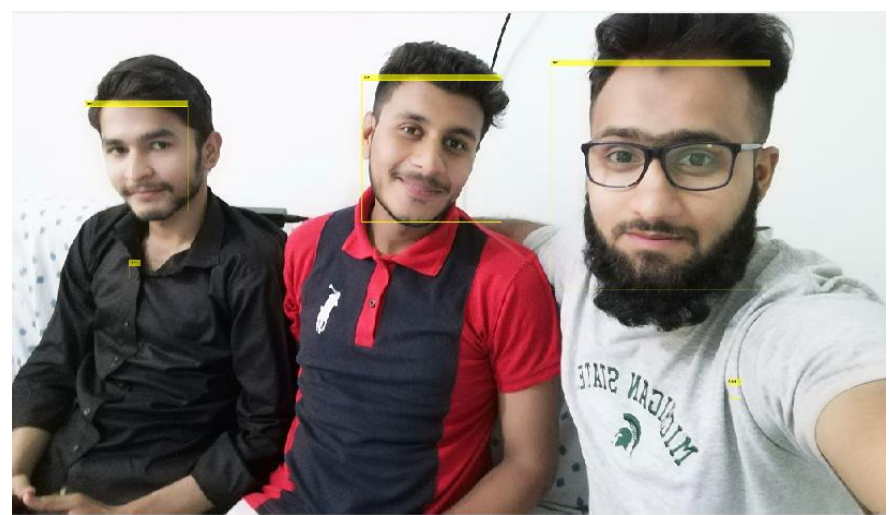

Fig. 5. Face detection. 


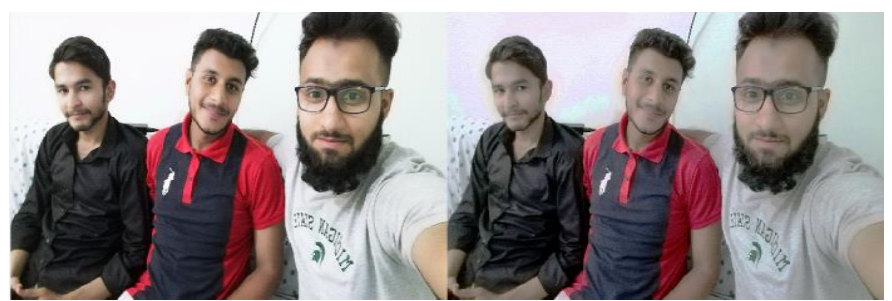

Fig. 6. Original image vs Equalized image.
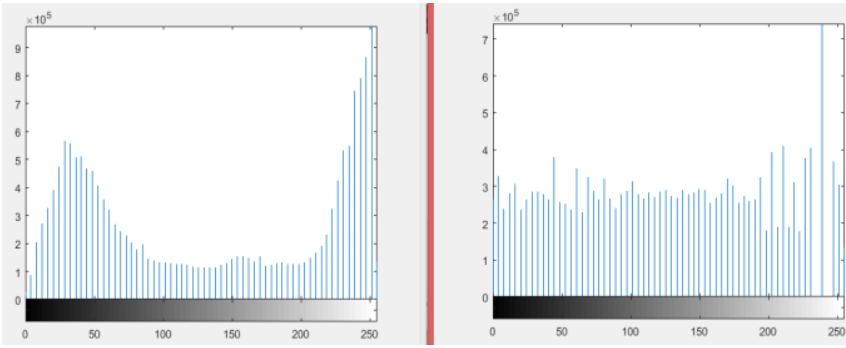

Fig. 7. Histogram plotting on original image vs Equalized image.
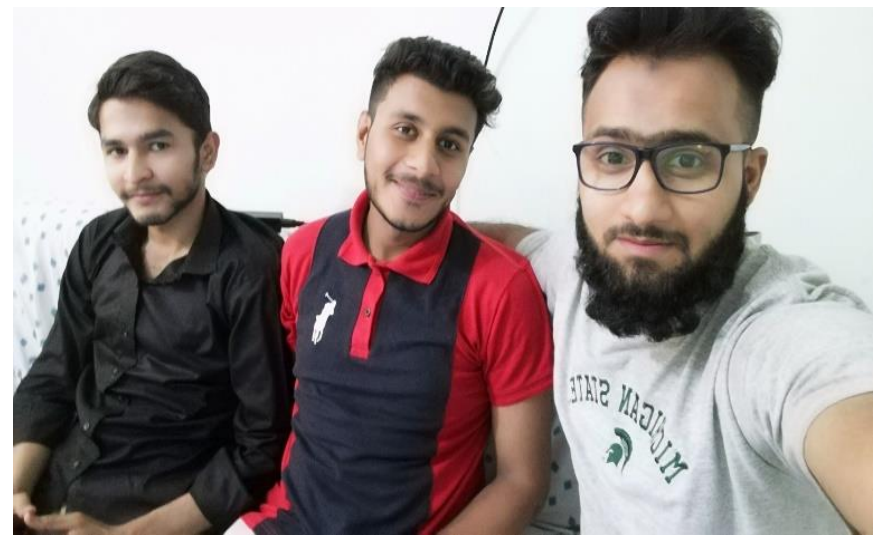

Fig. 8. Original image.
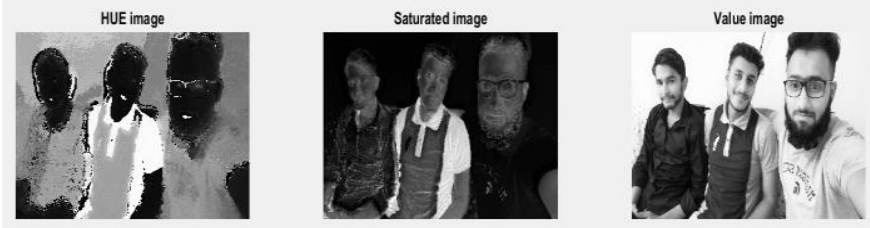

Fig. 9. Hue saturation value (HSV) of original image.
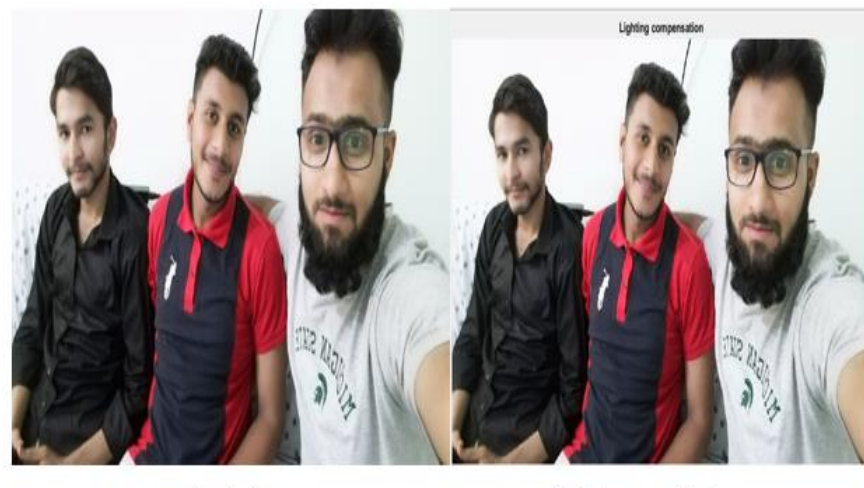

Original image

Light compensation image

Fig. 10. Light compensation from original image.

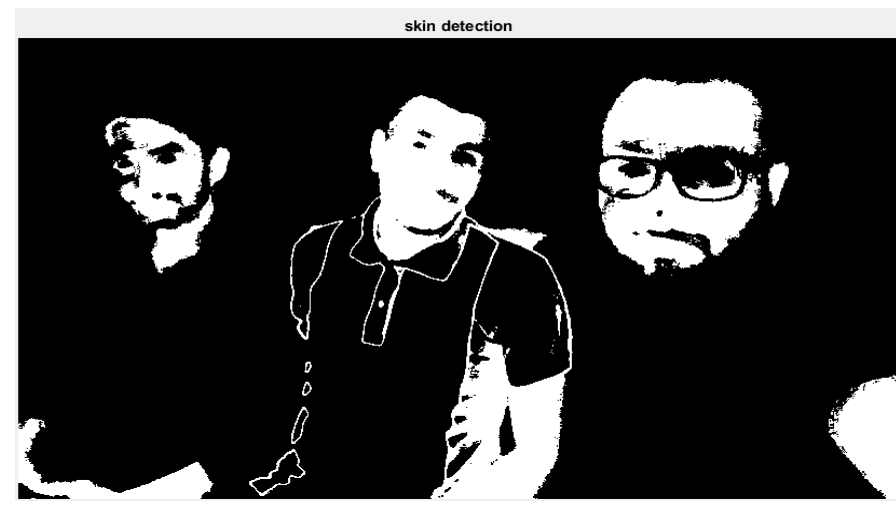

Fig. 11. Skin detection with NOGIE.

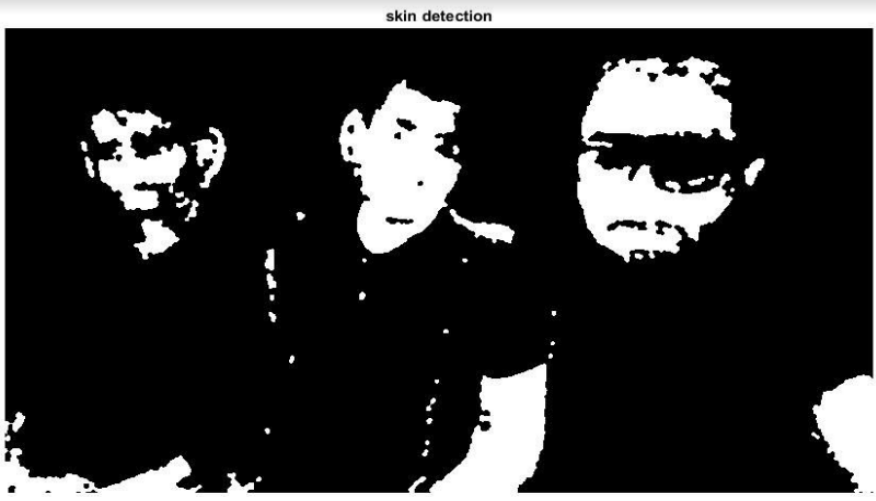

Fig. 12. Skin detection with NOWGIE.

\section{CONCLUSION}

In the digital image processing field, facial recognition or finger print recognition are a part of the skin detection topic. Although there are multiple skin detection techniques applied but the proposed methodology in this article have provided very satisfying results, the methodology implies on the HSV that gives more better output in than RGB or YCbCr. The proposed methodology comprises of the object detection technique which enables it to only detect human skin from the input comprising of the existence of any material. The mentioned algorithms NOGIE and NOWGIE, both of these algorithms are applied on the same input providing different results but they are compared for the better understanding of the methodology. NOWGIE provides more better results in comparison to NOGIE. Due to special image enhancement by the equalization technique is applied on the input in NOWGIE that is not applied in NOGIE but in both algorithms noise is removed that gives improved skin detection this enhanced version of the previous methodology gives far more better and improved results as this methodology comprises of the object detection techniques by which it first detects the human skin that acts as an object apart from any other skin. The skin pixel ratio of this proposed method and previously proposed method are mentioned in Table I. In the with GIE and without GIE techniques the skin pixel values are different for old and new algorithms but when compared the skin pixel percentage in with GIE method is less than the without GIE due to the accuracy of detecting only skin pixels while without GIE is less accurate as it is detecting a bit of the non-skin pixels as well. 
TABLE I. ASPECT OF SKIN PIXEL Ratio IN NEW AND OLD METHODOLOGY

\begin{tabular}{|c|c|c|c|c|c|c|c|}
\hline \multirow{2}{*}{$\begin{array}{l}S \\
\mathbf{r} \\
\mathbf{n} \\
\mathbf{o}\end{array}$} & \multirow{2}{*}{$\begin{array}{l}\text { Algorit } \\
\text { hm }\end{array}$} & \multicolumn{3}{|c|}{ With GIE } & \multicolumn{3}{|c|}{ Without GIE } \\
\hline & & $\begin{array}{l}\text { Total } \\
\text { pixels }\end{array}$ & $\begin{array}{l}\text { Skin } \\
\text { pixels }\end{array}$ & $\begin{array}{l}\text { Skin } \\
\text { pixel } \\
\text { percent } \\
\text { age }\end{array}$ & $\begin{array}{l}\text { Total } \\
\text { pixels }\end{array}$ & $\begin{array}{l}\text { Skin } \\
\text { pixels }\end{array}$ & $\begin{array}{l}\text { Skin } \\
\text { pixel } \\
\text { percent } \\
\text { age }\end{array}$ \\
\hline 1 & old & $\begin{array}{l}60057 \\
60\end{array}$ & $\begin{array}{l}12236 \\
28\end{array}$ & $20.37 \%$ & $\begin{array}{l}60057 \\
60\end{array}$ & $\begin{array}{l}17142 \\
42\end{array}$ & $28.54 \%$ \\
\hline 2 & new & $\begin{array}{l}60057 \\
60\end{array}$ & $\begin{array}{l}85842 \\
1\end{array}$ & $14.29 \%$ & $\begin{array}{l}60057 \\
60\end{array}$ & $\begin{array}{l}99444 \\
5\end{array}$ & $16.55 \%$ \\
\hline
\end{tabular}

\section{DISCUSSION AND FUTURE WORK}

Skin detection is a difficult task to perform in the area of digital image processing due to the difference in the skin tone of humans from different regions around the world. Although the human skin tone can also resemble to other things too. With the successful and satisfying results of the with GIE technique presented in this methodology. In future this method can be established for the identification of various skin diseases mainly skin cancer.

\section{ACKNOWLEDGMENT}

We will like to acknowledge our respected teachers Miss Arfa Hassan and Mr. Adnan Khan who believed in us, gave us the opportunity and played a major role in our supervision, we thank them and pay gratitude for their unconditional support and efforts it would not had had been possible without both of you.

\section{REFERENCES}

[1] Bush, Idoko John, Abiyev, Rahib, Sallam Mal'aitah, Mohammad Khaleel, and Altiparmak, Hamit, "Integrated artificial intelligence algorithm for skin detection,” ITM Web Conf., vol. 16, p. 2004, 2018.

[2] A. A. Zaidan, H. A. Karim, N. N. Ahmad, G. M. Alam, and B. B. Zaidan, "A new hybrid module for skin detector using fuzzy inference system structure and explicit rules," Int. J. Phys. Sci., vol. 5, no. 13, pp. 2084-2097, 2010.

[3] S. I. Shaikh, "Fusion Technique for Human Skin Detection," vol. 4, no. 06, pp. 1083-1089, 2015.

[4] A. Bhatia, S. Srivastava, and A. Agarwal, "Face Detection Using Fuzzy Logic and Skin Color Segmentation in Images," 2010 3rd Int. Conf. Emerg. Trends Eng. Technol., pp. 225-228, 2010.

[5] A. Hassan, U. Tariq, A. Iqbal, and M. A. Khan, "Muhammad Adnan Khan 4," vol. 2, no. 1, pp. 85-95, 2016.

[6] E. Elbaşi, "Fuzzy logic-based scenario recognition from video sequences,” J. Appl. Res. Technol., vol. 11, no. 5, pp. 702-707, 2013.

[7] P. F. Processor, "ITEE Journal," vol. 1, no. 1, pp. 30-38, 2012.

[8] M. Rai, R. K. Yadav, and G. Sinha, "Algorithm for Human Skin Detection Using Fuzzy Logic," pp. 1-6.

[9] B. Poon, M. A. Amin, and H. Yan, "PCA Based Human Face Recognition with Improved Methods for Distorted Images due to Illumination and Color Background," IAENG Int. J. Comput. Sci., vol. 43, no. 3, pp. 277-283, 2016.

[10] H. El Khiyari and H. Wechsler, “Age Invariant Face Recognition Using Convolutional Neural Networks and Set Distances,” J. Inf. Secur., vol. 08, no. 03, pp. 174-185, 2017. 